\title{
A Comparison of O Spectral Types with Nonrotating and Rotating Evolutionary Models
}

\author{
Nolan R. Walborn \\ Space Telescope Science Institute, 3700 San Martin Drive, Baltimore, \\ MD 21218, USA \\ Daniel J. Lennon \\ Isaac Newton Group, Apartado 321, 38700 Santa Cruz de La Palma, \\ Canary Islands, Spain
}

\begin{abstract}
A calibration of the $\mathrm{O}$ spectral types and luminosity classes has been plotted onto evolutionary tracks and isochrones for both nonrotating models and models with an initial rotational velocity of $300 \mathrm{~km} \mathrm{sec}^{-1}$. Differences in the physical interpretation of the spectral types between the two cases have been investigated.
\end{abstract}

\section{Data Sources and Procedures}

For the calibration of the spectral types and luminosity classes, we start with the $M_{V}$ values from Walborn (1973), supplemented by Walborn et al. (2002) for type O3. The adopted effective temperatures reflect the recently revised scale from line-blanketed model atmospheres, derived by Martins et al. (2002) for the main sequence, and by Crowther et al. (2002) and Herrero et al. (2002) for supergiants. The values for giants are simply means of the main-sequence and supergiant determinations. The bolometric corrections are from Vacca et al. (1996) for a given $T_{\text {eff }}$, not spectral type. The nonrotating evolutionary models are those of Schaller et al. (1992) for solar metallicity; note that they include convective overshooting. The rotating models are those of Meynet \& Maeder (2000) for an initial rotation of $300 \mathrm{~km} \mathrm{sec}^{-1}$.

The data are plotted in Figure 1 for the nonrotating case and in Figure 2 for the rotating case. In each case, the same calibration sequences are shown by solid lines labeled with spectral types for luminosity classes V, III, and Ia. The Humphreys-Davidson Limit is shown by a bold, solid line segment at upper right. The evolutionary tracks are shown by dashed lines labeled with $M / M_{\odot}$ at their ZAMS initial points. All tracks are truncated at the right border of the figures, i.e., portions returning from lower temperatures are not shown; the latter evolutionary stages are rapid and are not believed to correspond to $\mathrm{O}$ spectral types. Isochrones for $\log$ ages of $6.2,6.4,6.6$, and $6.8(1.6,2.5,4.0$, and $6.3 \mathrm{Myr}$, respectively) are shown as dotted lines. 


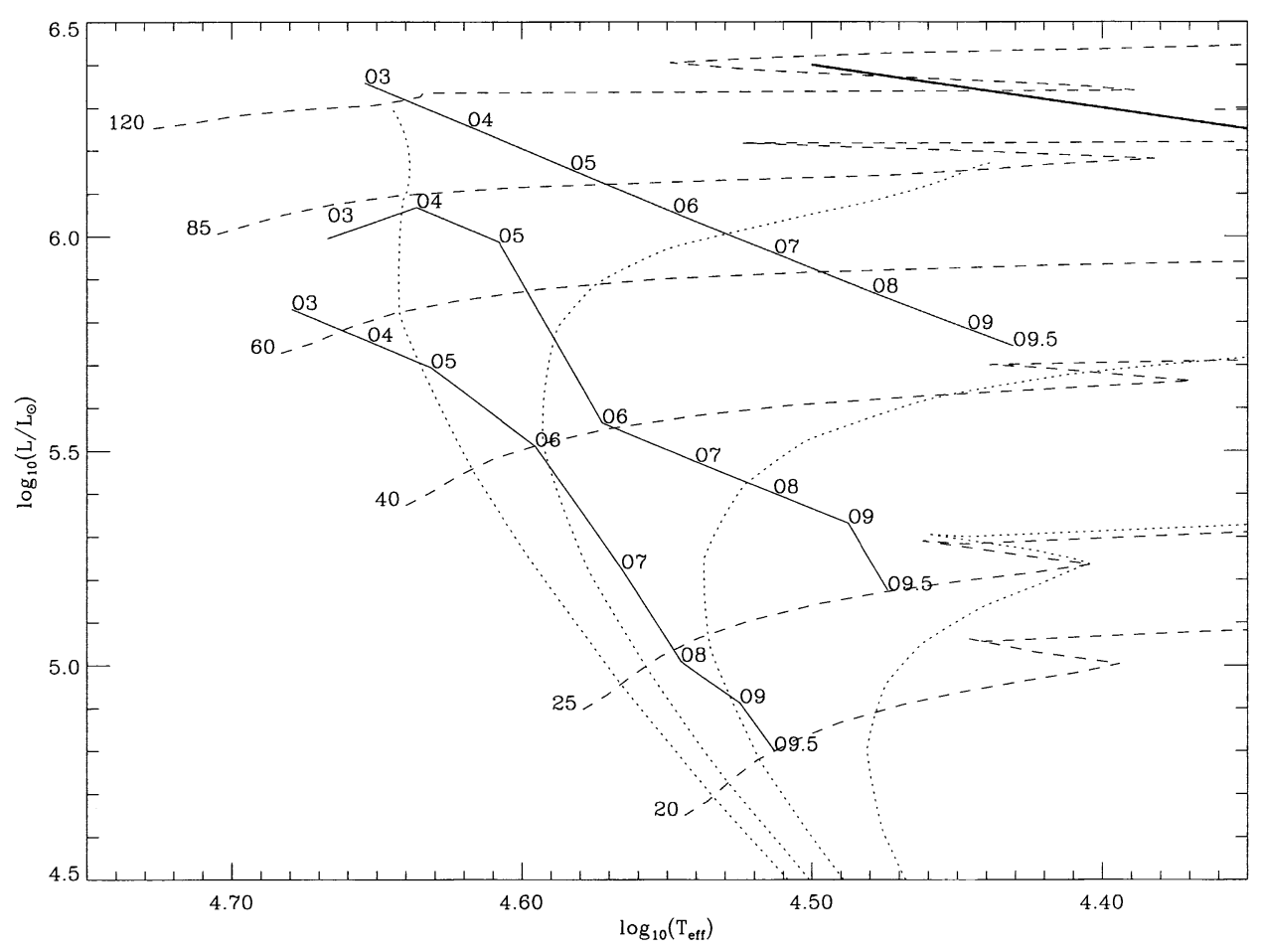

Figure 1. Nonrotating models

\section{Results}

The following conclusions can be drawn from analysis of the figures.

- Typical luminosity class V stars are evolved some distance off the ZAMS, although the earliest types are seen to be closer.

- There is a gap between the supergiant sequence and the Humphreys-Davidson Limit, which is presumably the domain of WNL, LBV, and related objects.

- The unpopulated region at upper left may be the realm of the as yet uncalibrated O2 stars (Walborn et al. 2002).

- Mass differences at a given spectral type between the nonrotating and rotating cases are not significant, being comparable to the observational errors in their determination and the differences between different sets of theoretical tracks.

- The physical main sequence (i.e., ZAMS to hydrogen exhaustion at the kinks or reversals in the tracks) is substantially more compressed in $T_{\text {eff }}$ in the rotating case, but the absolute main-sequence lifetimes are similar in the two cases.

- The absolute ages of luminosity class $\mathrm{V}$ are also not significantly different between the two cases.

- All O spectral types are burning hydrogen in the core in the nonrotating case.

- In contrast, the rotating giants are near the hydrogen-exhaustion locus, that is, their evolutionary ages are far more advanced than those of the nonrotating giants, and their absolute ages are about $15 \%$ greater on the average. Note that 


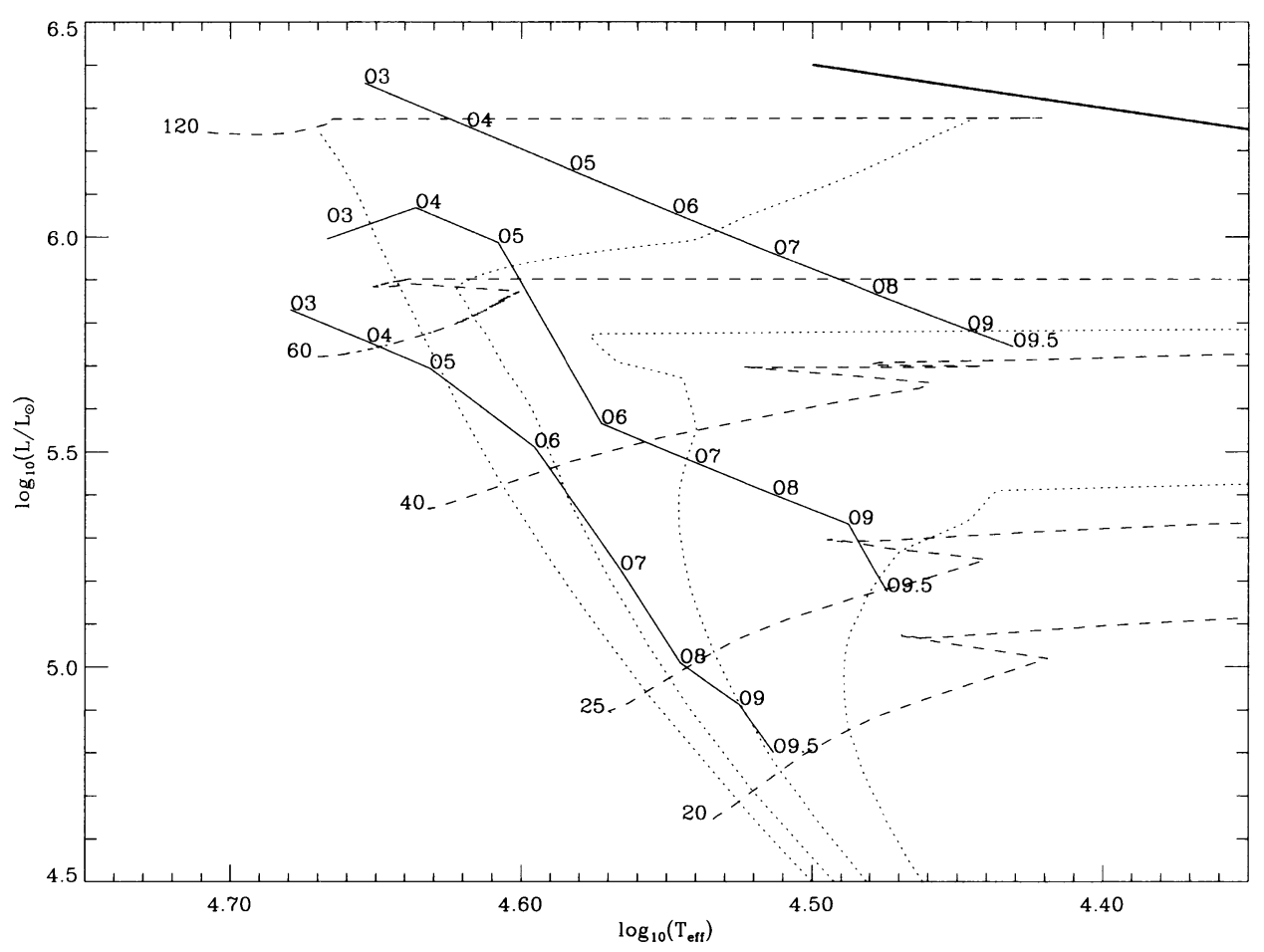

Figure 2. Initial rotational velocity $300 \mathrm{~km} \mathrm{sec}^{-1}$

luminosity classes II and Ib are first distinguished at spectral type O6.

- The rotating supergiants are post-hydrogen burning and their absolute ages are about $10 \%$ greater than those of the nonrotating counterparts. Since those stages have rapid timescales and are suggested to correspond to Wolf-Rayet spectra (Meynet \& Maeder 2000), the implication is that O-type supergiants may derive only from initially slow rotators.

Acknowledgments. NRW's travel to Cancun was supported by NASA through grant GO-7437.04-96 from STScI, which is operated by AURA, Inc., under contract NAS5-26555.

\section{References}

Crowther, P.A. et al. 2002, ApJ, 579, 774

Herrero, A., Puls, J., \& Najarro, F. 2002, A\&A, 396, 949

Martins, F., Schaerer, D., \& Hillier, D.J. 2002, A\&A, 382, 999

Meynet, G., \& Maeder, A. 2000, A\&A, 361, 101

Schaller, G., Schaerer, D., Meynet, G., \& Maeder, A. 1992, A\&AS, 96, 269

Vacca, W.D., Garmany, C.D., \& Shull, J.M. 1996, ApJ, 460, 914

Walborn, N.R. 1973, AJ, 78, 1067

Walborn, N.R. et al. 2002, AJ, 123, 2754 